\title{
Femto Cells: Current Status and Future Directions
}

\author{
Khaled Elleithy and Varun Rao \\ Department of Computer Science and Engineering, \\ University of Bridgeport, Bridgeport, CT, USA 06604 \\ elleithy@bridgeport.edu , varunrao@bridgeport.edu
}

\begin{abstract}
This is a survey paper on the recently developed and rapidly evolving field of femtocells. Quite often, it is noticed that cell-phone signals are strongly attenuated, when indoors, leading to call dropping or poor call quality. Femtocells are mini base stations that are deployed in users' homes so that the user can directly connect to the cellular network through the femtocell instead of the outdoor macrocell, thereby increasing call quality. In the later stages of the paper, we also discuss the integration of the femtocell into the $3 G$ architecture, as well as the various interference issues that the femtocell faces.
\end{abstract}

\section{Keywords}

Femtocell, UMTS, Revised UMTS architecture

\section{Introduction}

The last few years have seen tremendous growth in the fields of wireless networks and telecommunications. There are over four billion mobile phone users in the world today, and the numbers continue to rise. However, cellular phones continue to face issues such as poor signal strength and call quality when used indoors. At the same time, there has also been a huge development in Voice over IP (VoIP) applications. This technology allows users to make free calls through the internet, thereby acting as a potential threat to mobile operators around the world.

In order to ensure customer loyalty and satisfaction, with a view to improving signal-strength in restricted areas, mobile operators needed to come up with an efficient solution. The deployment of femtocells was one such solution. Femtocells ${ }^{[1]}$ are small base stations installed in homes. They are similar in size to a router and offer excellent signal coverage indoors, thereby reducing the load on the external macrocell. In order to avail the features of a femtocell, a user must have an internet broadband connection. The user must then, purchase the femtocell from a mobile operator and simply plug it to the connection. It is imperative that a femtocell remains a simple plug-and-play device, as a complex installation process is likely to prevent users from adopting it. When the user enters their home, the femtocell will detect the mobile handset and vice versa, and a connection will be established. All calls are then made via the femtocell. This technology is being tested by mobile operators around the world and is thought to be the technology that will revolutionize cellular communication around the world.

However, unlike Wi-Fi, femtocells operate in the licensed spectrum. In most countries, mobile operators are allotted three, licensed $5 \mathrm{MHz}$ frequency bands ${ }^{[2]}$. In order to maintain customer

DOI : 10.5121/ijngn.2011.3101 
satisfaction and maximize profits, operators need to utilize these bands intelligently and efficiently. Since both femtocells and macrocells are required to operate in these limited bandwidths, there are bound to be interference issues. A few proposed solutions that can help alleviate these drawbacks are seen in the later part of the paper.

\section{Implementing the femtocell}

\subsection{Operation of the femtocell}

As mentioned in the previous section, a broadband internet connection is a prerequisite for connecting a femtocell. The femtocell enables encryption for all voice calls and data sent or received by the mobile phone. This makes it impossible for an external user to break into a user's home network. To a standard $3 \mathrm{G}$ cellular phone, the femtocell appears as another cellsite or macrocell, hence communicating with it as it would with a macrocell, when the mobile phone is used outdoors. Since femtocells operate at very low radio power levels, battery life is high. Also, as the distance between the femtocell and the mobile handset is short, call quality is excellent.

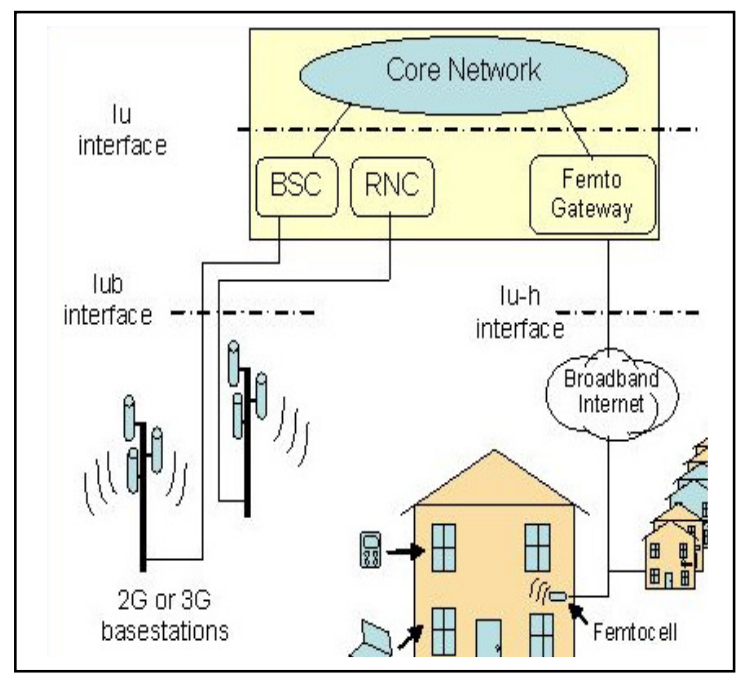

Figure 1. Femtocell deployment ${ }^{[3]}$

The mobile operator's telephone switch and data switch communicate with the femtocell gateway in the same way as for other mobile calls. Therefore, all services including phone numbers, call diversion, voicemail etc. all operate in exactly the same way and appear the same to the end user

The connection between the femtocell and the femtocell gateway is encrypted using IPSec, which prevents interception. There is also authentication when the femtocell is installed for the first time to ensure that the access point is a valid one. Inside the femtocell are the complete workings of a mobile phone basestation. Additional functions are also included, such as some of the RNC ${ }^{[8]}$ (Radio Network Controller) processing, which would normally reside at the mobile switching centre. Some femtocells also include core network element so that data sessions can be managed locally without needing to flow back through the operator's switching centres. 
The extra capabilities of a femtocell demand it to be self-installing and self-configuring. This requires considerable extra software which scans the environment to determine the available frequencies, power level and/or scrambling codes to be used, thereby increasing complexity to a certain extent. This is a continuous process to adapt to changing radio conditions.

\subsection{Femtocell radio technologies}

The most commonly used implementation of the femtocell, makes use of the $3 \mathrm{G}^{[5]}$ UMTS standard. However, other radio technologies are also being tested with femtocells and could be successfully launched in the near future. The most commonly used radio technologies ${ }^{[5]}$ are as below:

\section{a. GSM}

The most commonly used wireless technology, GSM accounts for $85 \%$ of the current mobile market share. GSM cellsites are termed as picocells rather than femtocells because they are not auto-configuring. They require the operator to get these cellsites up and running for use.

\section{b. UMTS}

This technology is an evolution of GSM; hence it is also known as 3G. It was derived from GSM by replacing the standard GSM radio sub-system, with one based on the CDMA technique. It offers a much larger capacity as compared to GSM and also requires a lesser number of cellsites. UMTS networks are usually used in combination with GSM technologies.

\section{c. High Speed Packet Access (HSPA)}

This is an improved version of UMTS obtained by increasing coding on radio transmissions, thereby improving throughput to a large extent. They provide data rates of upto $21 \mathrm{Mbits} / \mathrm{sec}$. They work satisfactorily with UMTS equipment. However, new handsets would be required to take advantage of the high data rates provided by HSPA.

\section{d. Code Division Multiple Access (CDMA)}

This standard grew in popularity at its launch but did not achieve the global assimilation that was expected of it. The first phase of CDMA was termed '1xRTT', an efficient technology for voice and text services.

\section{e. Long Term Evolution (LTE)}

This is a joint undertaking by GSM and CDMA vendors in order to develop a common standard for mobile communications. This is a $4 \mathrm{G}$ standard and is capable of achieving data transfer rates of upto 100 Megabits per second. It uses the orthogonal frequency division multiplexing (OFDM) scheme, in order to tackle issues such as multi-path propagation. 


\section{f. WiMAX ${ }^{[6]}$}

This is a standard that is used to provide wireless broadband services in regions where it is infeasible to set up fixed telephone systems. It makes use of OFDM technology and is the biggest competitor to the

LTE system mentioned above. It supports data rates of upto 75 Megabits per channel, making it an excellent alternative for femtocell implementation.

\section{Femtocell integration into the UMTS architecture}

UMTS $^{[7]}$ is a third generation $(3 \mathrm{G})$ mobile communications technology which is also being developed into 4G. Many solutions have been proposed in order to provide a smooth transition from the second generation GSM system to a third generation. Therefore, UMTS is sometimes marketed as 3GSM, emphasizing the close relationship with GSM and differentiating it from competing technologies.

The UMTS architecture ${ }^{[7]}$ is composed of three major domains: User Equipment, UTRAN and the Core Network.

\section{a. User equipment (UE)}

The user equipment domain is assigned to a single user. It consists of functions that are required for the user to access the various UMTS services. This domain includes User Subscriber Identity Module (USIM) and mobile equipment domain. The Subscriber Identity Module (SIM) contained within the USIM, performs functions related to encryption and user authentication. The user equipment also consists of the actual end device itself. All necessary user interfaces and radio transmission modules are located here.

\section{b. UTRAN}

The UTRAN consists of the various radio interfaces and also handles cell level mobility. It comprises several radio network sub-systems (RNS). A NodeB is used to control several antennae, making a radio cell. The UTRAN is connected to the user equipment via the $U_{u}$ interface and to the core network via the $\mathrm{I}_{\mathrm{u}}$ interface. Each RNS is controlled by a radio network controller (RNC). The functions of the UTRAN include

- congestion control

- encryption and decryption

- code allocation

- handover control

- management

\section{c. Core Network $(\mathrm{CN})$}

The core network contains functions for inter-system handover, gateways to other networks (fixed and wireless). It also performs location management if there is no dedicated connection between UE and UTRAN. The core network is a combination of circuit-switching and packet- 
switching elements. The circuit-switched elements include GSM components such as mobile services switching center (MSC), gateway MSC (GMSC) and Visitor Location Register (VLR). The packet-switched elements include GPRS components such as gateway GPRS support node (GGSN) and serving GPRS support node (SGSN).

Thus, the infrastructure of UMTS enables cellular operators using GSM, to switch over easily to UMTS since there is reuse of components, thereby saving money and resources. However, the standard UMTS architecture cannot sustain the addition of a femtocell. There are a few modifications made to the standard UMTS architecture in order to accommodate the integration of a femtocell. The new essential network elements in the revised UMTS architecture ${ }^{[10]}$ are the femtocell, itself, known here as the $3 \mathrm{G}$ Home NodeB (HNB), the $3 \mathrm{G}$ Home NodeB Gateway (HNB GW), Security Gateway (SeGW) and the 3G Home NodeB Management System (HMS).

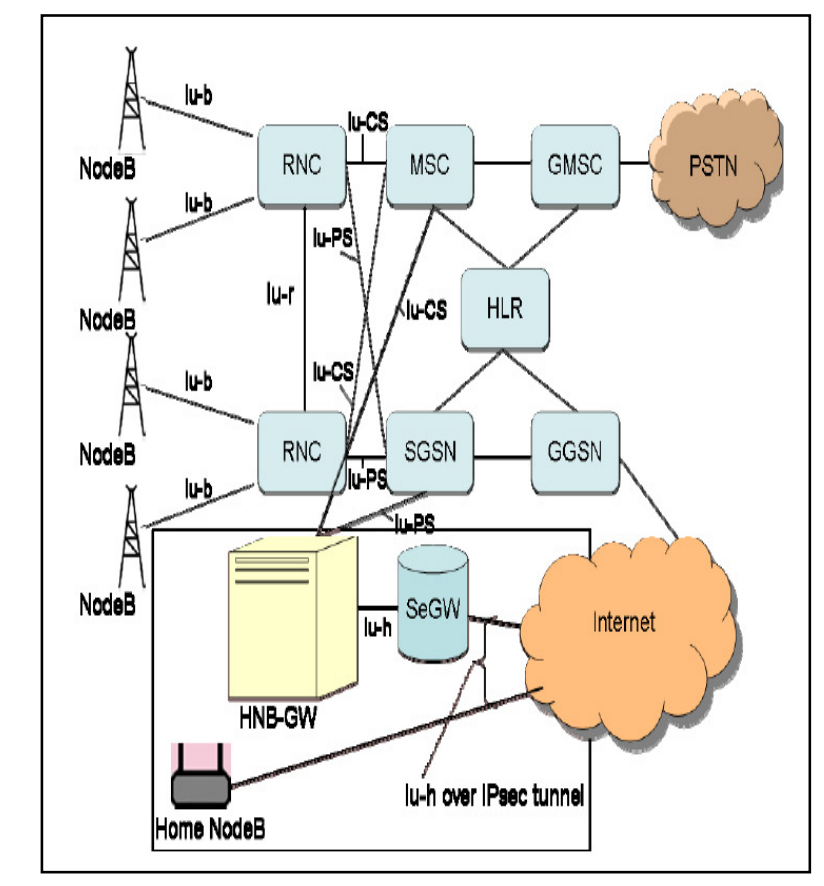

Figure 2. Femtocell in UMTS architecture ${ }^{[10]}$

\section{- Home NodeB}

The $3 \mathrm{G}$ Home NodeB (HNB) is the device that is installed in the user's premises, serving as a femtocell. The HNB is able to operate with 4 to 8 existing UEs and offer them the same services as if they were operating under a regular NodeB. The device is low cost and relatively small in size and can be installed in the user's home, office or a location he/she chooses. The operator has no exact control of the location. The HNB is powered from the user's electric network using most likely, an external power adapter. 


\section{- Home NodeB Gateway}

The Home NodeB Gateway (HNB-GW) is the device used to connect the HNBs to the UMTS network. The HNB-GW concentrates connections from a large amount of femtocells. The new $\mathrm{I}_{\mathrm{u}^{-}}$ $\mathrm{h}$ interface is used between HNB and HNB-GW. HNB-GW is connected to the CN using the standard $\mathrm{I}_{\mathrm{u}}$ interface and the network sees it as a standard RNC. The HNB-GW can be located anywhere at the operator's premises.

\section{- Security Gateway}

The Security Gateway (SeGW) is a logical element which can be physically implemented separately or as an integrated solution with the HNB-GW. SeGW acts as a firewall between the operator's core network elements and the public internet.

\section{- Home NodeB Management System}

The Home NodeB Management System (HMS) uses an interface based on the TR-069 standards widely used in DSL modem and DVB set-top-box management and updates. The management system sends the configuration data to the HNB and helps the HNB in HNB-GW and SeGW discovery. It can also initiate HNB software updates and perform HNB location verification.

\section{Interference issues in femtocells and interference mitigation}

Although the growth of the femtocell could see a sharp rise in the popularity of cellular phones, there are still concerns regarding interference between femtocells and the external macrocell as well as similar such devices. Since the femtocell and the macrocell operate in the same range of frequencies, there is bound to be interference. The main problem of interference arises from the fact that femtocells are installed in an ad-hoc manner, or independent of the structure of the cellular network. One simple solution discussed, was for the femtocell to operate on a different carrier frequency with respect to the macrocell. However, this solution is not simple to implement since most mobile operators are allotted a fixed spectrum to provide their services, as mentioned earlier. Another effective solution that successfully tackles femtocell interference for outdoor mobile users connected to the macrocell is suggested in [15]. To find an effective and logical solution to this drawback, the Femto-forum ${ }^{[13]}$ has been involved in conducting research into mitigating the problem of interference in femtocells.

In the $3 \mathrm{G}$ architecture, most operators are allotted 2 frequencies for use. So, interference could also be reduced by allowing femtocells to transmit on the unused second frequency. However, this still does not resolve interference that may arise between two femtocells that operate on, say two floors of a building. This scenario may also affect the quality of service and capacity of each individual femtocell. Another alternative suggested, was for mobile operators to implement a two-tier femtocell network ${ }^{[2]}$, by sharing spectrum rather than splitting spectrum between the two tiers.

As per studies conducted by the Femto-forum ${ }^{[13]}$, the main forms of interference that arises in femtocells are mentioned below: 
- Femtocells interfering with base-stations on the same frequency: a large number of femtocells operating on the same carrier frequency may increase the load on the overlaying macrocell, thereby reducing the capacity of the entire network.

- Base stations interfering with femtocells on the same frequency: this scenario is identical to the one mentioned above.

- Femtocells interfering with each other: if there are multiple femtocells operating close to each other, they produce a level of background noise that reduces the quality of service of each femtocell.

- Cell phone signals received by both, macrocells and femtocells: In some cases, when a mobile phone connects to a femtocell, the signal strength is strong enough to be received by the surrounding macrocell as well. This leads to a level of noise at the macrocell, which are beyond its tolerable level.

All the scenarios mentioned above, can lead to degradation of the capacity of the overall network, thereby reducing the quality of service offered by the network.

On account of the different types of interferences that arise in femtocells, different techniques must be implemented in order to bring down interference to acceptable levels. One such technique has been suggested in [4]. In this paper, Chandrashekhar and Andrews have developed an architecture that helps in limiting cross-tier interference between femtocells and macrocells. This helps in increasing the uplink capacity for a shared spectrum network. A few more proposed fixes ${ }^{[15]}$ to help reduce femtocell interference are as below:

- Adaptive Pilot Power control: the femtocell detects transmission signals from other devices around it and accordingly adjusts its own transmission signal power while still maintaining its coverage area.

- Dynamic receiver gain management: for satisfactory operation, there must be some sort of automatic gain or attenuation adjustment in femtocells. This will enable the user equipment to operate without increasing their transmitting power beyond a certain extent, thereby keeping the levels of noise and interference to a minimum.

- Mobile phone uplink power capping: this technique places a limit on the maximum power output of the mobile phone in the femtocell environment. This allows the mobile phone to perform a hand-over ${ }^{[14]}$ to the macro network without increasing the transmission power to a level where it adds noise to the macro network.

- Extended dynamic range for femtocell receiver: In order to ensure that the femtocell operates with maximum efficiency in the presence of high-power mobile phones connected to the macrocell, its receiver must have a very high dynamic range.

\section{Conclusion}

The promising femtocell is being tested extensively by mobile operators around the world. However, there are still some issues that need to be worked on for femtocells to be implemented as fault-free devices. In the years to come, femtocells may also be able to operate efficiently using EDGE ${ }^{[5]}$ standards. A number of hardware evolutions are required before high usability and quality of service standards are achieved. This may take a few years to achieve. Mobile operators 
must continue partnering with internet service providers, so as to make the femtocell a reasonable means of improving cellular communication indoors. There is still sufficient capacity available in the macro network, so there is still no immediate need of femtocells to help alleviate the pressure on macrocells. However, femtocells can be of immense help in rural areas where the distances between homes and the nearest macrocell, could be many miles. The development of femtocells can also help speed up the evolution of Universal Mobile Access ${ }^{[16]}$ (UMA).

\section{References}

1. Haddad, Y.; Porrat, D.; Femtocell: Opportunities and challenges of the home cellular base station for 3G; Proceedings of IEEE Global Telecommunications Conference, 2007. Washington DC, USA, pp. 3317-3321.

2. Choi D, Monajemi P, Kang S, Villasenor J; Dealing with Loud Neighbors: The benefits and Tradeoffs of Adaptive Femtocell Access; IEEE Global Telecommunications Conference, 2008.

3. Chandrasekhar V, Andrews J, Gatherer A; Femtocell networks: a survey; IEEE Communications Magazine, Volume 46, 2008.

4. Chandrasekhar V. and Andrews J.; Uplink Capacity \& Interference Avoidance for Two-Tier Networks; Proceedings of IEEE Global Telecommunications Conference, 2007. Washington DC, USA; Pages 3322-3326.

5. Rao Y.S., Wing-Cheong Yeung, Kripalani A.; Third generation (3G) Radio Access Standards; International Conference on Communication Technology Proceedings, Beijing; $21^{\text {st }}$ August 2000 - $25^{\text {th }}$ Aug 2000; Volume 2, pages $1017-1023$.

6. Shu-ping Yeh, Talwar S., Seong-choon Lee, Heechang Kim; WiMAX femtocells: a perspective on network architecture, capacity and coverage; IEEE Communications Magazine; Issue Date: October 2008; Volume 46, Issue 10; Pages 58 - 65.

7. Schiller J; Mobile Communications; pages 93-164; $2^{\text {nd }}$ Edition; ISBN 0-321-12381-6.

8. Hasan, S.F.; Siddique, N.H.; Chakraborty, S.; Femtocell vs. WiFi - A survey and comparison of architecture and performance; 1st International Conference on Wireless Communication, Vehicular Technology, Information Theory and Aerospace \& Electronic Systems Technology, 2009. Publication Year: 2009, Page(s): 916 - 920.

9. Edwards, C; The future is femto; IET journal, Volume: 3, Issue: 15; Publication Year: 2008, Page(s): 70 - 73.

10. Neruda, M., Vrana, J, Bestak, R; Femtocells in $3 G$ mobile networks; $16^{\text {th }}$ International conference on systems, signals and image processing, Chalkida; Publication year: $18^{\text {th }}-20^{\text {th }}$ June, 2009; pages $1-4$.

11. Holma H, Toskala A; HSDPA/HSUPA for UMTS; John Wiley \& Sons 2006; ISBN 0-470-018844.

12. Holma H, Toskala A; WCDMA for UMTS; John Wiley \& Sons 2002, ISBN 978-0470844670. 
International Journal of Next-Generation Networks (IJNGN) Vol.3, No.1, March 2011

13. Femto Forum; Interference Management in UMTS Femtocells; 2008, available online at www.femtoforum.org

14. Chowdhury M.Z., Ryu W., Rhee E., Jang Y. M.; Handover between femtocell and macrocell for UMTS-based networks; $11^{\text {th }}$ International Conference on Advanced Communication Technology, 2009; Issue date: 15-18 Feb, 2009; Volume 1, Pages 237-241.

15. Han K., Choi Y., Kim D., Na M., Choi S., Han K.; Optimization of femtocell network configuration under interference constraints; 7th International Symposium on Modeling and Optimization in Mobile, Ad Hoc, and Wireless Networks, 2009; Issue Date : 23-27 June 2009; Pages 1-7.

16. Arjona, A., Verkasalo, H; Unlicensed Mobile Access Handover and Packet Data Performance Analysis; Second International Conference on Digital telecommunications, 2007. ICDT '07; Issue Date: $1^{\text {st }}-5^{\text {th }}$ July, 2007; Pages: 9-9.

\section{Authors}

Khaled Elleithy is the Associate Dean for Graduate Studies in the School of Engineering at the University of Bridgeport. He has research interests are in the areas of network security, mobile communications, and formal approaches for design and verification. He has published more than one hundred twenty research papers in international journals and conferences in his areas of expertise. Dr. Elleithy is the co-chair of the International Joint Conferences on Computer, Information, and Systems Sciences, and Engineering (CISSE). CISSE is the first Engineering/Computing and Systems Research EConference in the world to be completely conducted online in real-time via the internet and was successfully running for four years.

Dr. Elleithy is the editor or co-editor of 10 books published by Springer for advances on Innovations and Advanced Techniques in Systems, Computing Sciences and Software.

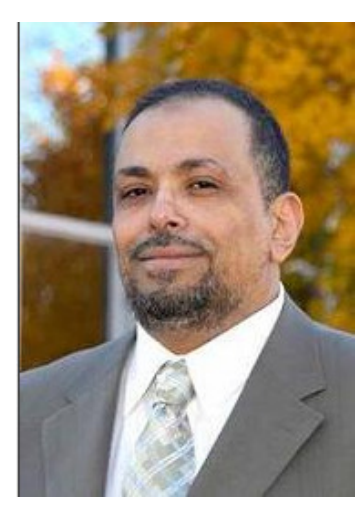

Varun Rao is a graduate student of Computer Science at the University of Bridgeport. He received his Bachelor's degree in Computer Engineering from the University of Mumbai, India. His research interests include next-generation networks, mobile communications and $3 \mathrm{G}$ and $4 \mathrm{G}$ wireless technologies. 